\title{
A Cross-layer Approach for Packet Scheduling in Reliable Multicast Data Transmission over Geostationary Satellite Networks
}

\author{
A. Sali, G. Acar, B. Evans \\ Center for Communication Systems Research (CCSR), \\ University of Surrey, \\ GU2 7XH Guildford, \\ United Kingdom.
}

\begin{abstract}
In this paper, we propose a channel-aware scheduling algorithm that exploits the reported Channel State Information (CSI) from all users in the multicast group for reliable transmission of multicast information over a geostationary satellite network. Reliability is guaranteed via a multicast transport protocol that retransmits lost segments to the multicast group. The proposed scheduling mechanism uses cross-layer CSI before making a decision whether or not a data segment is to be transmitted. As such, the algorithm avoids unfavourable channel conditions to reduce the forward link resources that would be wasted to retransmit lost segments. Scheduling delay and retransmission delay are found to be the elements of a trade-off, and simulations are conducted to attain optimal algorithm parameters to minimize session transfer delay in the face of L-band mobile channel conditions.
\end{abstract}

Index Terms - cross-layer design, channel-state-information, packet scheduling, geostationary satellite networks

\section{INTRODUCTION}

$\mathrm{R}$ esearch on cross-layer design has recently attracted significant interest. Cross-layer design suggests (possibly joint) adaptation of protocol mechanisms at various layers according to the information collected at other layers of the communication system. Recent growth of heterogeneous networks entails adaptive mechanisms. In this frame, a cross layer approach would be more effective and flexible. In wireless systems where both radio resources and power are strictly constrained, resource optimisation is needed when such optimisation is not guaranteed by the current layered protocol stack. For an instance, in [1], to achieve efficient resource allocation, channel variations and traffic burstiness information are exploited resulting significant increase in resource utilisation. In [2], the scheduling information in medium access control (MAC) layer is merged with rate selection in physical/link (PHY/LINK) layer. The mechanism interestingly relies on a low-complexity 1-bit ACK/NACK feedback signal from receivers indicating the correct reception/failure of the segment in downlink transmission. In [3], cross-layer design for multiuser scheduling at the data link layer is developed where each user employs adaptive modulation and coding (AMC) scheme at PHY layer.

\section{Multicast TRANSMISSION OVER SATELLITE NETWORKS}

In transmission of multicast data from a satellite to $N$ direct receivers, repeated retransmission process due to bad channel condition may exhaust the forward link capacity. This problem is exacerbated for large multicast groups. In order to countermeasure this problem, we propose a channel-aware packet scheduling algorithm on the forward link that exploits time-varying channel variations to reduce the number of retransmissions.

The reference system in this study is based on a L-Band geostationary satellite network similar to Inmarsat BGAN (Broadband Global Area Network) TDMA-based system as depicted in Figure 1. The packet scheduler is at the Radio Network Controller (RNC) and a geostationary satellite relays the multicast information to all multicast users through Multicast Terminals (MT) and Terminal Equipment (TE).

We assume that multicast reliability is achieved via a multicast transport protocol with a retransmission strategy that is based on StarBurst MFTP (Multicast File Transfer Protocol) [5]. In the reference model, the satellite-receivers links form one-tomany (star-based) reliable multicast protocol. More specifically, through this higher layer protocol, we assume that a multicast file is transmitted to all multicast group members in its entirety. In response, the receivers send feedback messages for lost segments. Subsequent passes may be initiated by the multicast server to retransmit the lost segments. Note that MFTP allows retransmissions of lost segments only after the end of current pass of segments. 


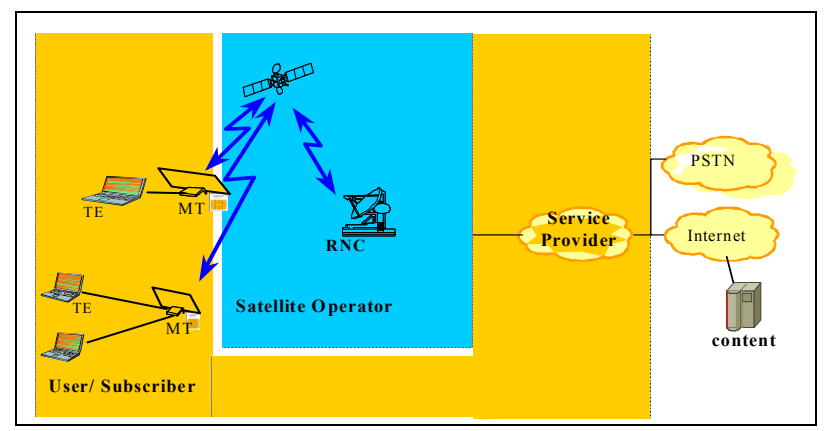

Figure 1 Reference system architecture

\section{CHANNEL MODEL}

We consider a two-state semi-Markov model [4], that alternates between a 'good' state representing LOS (Line-OfSight) areas and a 'bad' state representing shadowed areas. The sojourn time spent in the LOS state follows power law distribution. The duration in the shadowed state follows a lognormal distribution. The semi-Markov model parameters are displayed in Table I.

\begin{tabular}{|c|c|c|c|c|c|c|c|c|}
\hline \multirow{2}{*}{$\begin{array}{c}\text { Prop. } \\
\text { Type }\end{array}$} & \multirow{k}{*}{$\boldsymbol{k}$} & \multicolumn{2}{|c|}{$\boldsymbol{\mu}$} & \multirow{2}{*}{ Open (LOS) } & \multicolumn{2}{|c|}{ Shadowed } \\
\cline { 3 - 4 } & & $\boldsymbol{P}_{\boldsymbol{0 1}}$ & $\boldsymbol{P}_{\boldsymbol{0} \mathbf{2}}$ & $\boldsymbol{\sigma}$ & $\boldsymbol{\beta}$ & $\boldsymbol{\chi}$ & $\boldsymbol{\alpha}$ & $\boldsymbol{\delta}$ \\
\hline 1 & 2.00 & 3.50 & 3.00 & 2.7 & 0.88 & 0.61 & 2.68 & 1.45 \\
\hline 2 & 2.00 & 2.75 & 4.00 & 3.1 & 0.21 & 0.58 & 0.22 & 0.12 \\
\hline 3 & 5.01 & 3.25 & 1.75 & 0.8 & 0.12 & 0.84 & 0.22 & 1.22 \\
\hline 4 & 1.00 & 3.00 & 4.00 & 0.4 & 0.83 & 0.66 & 3.14 & 1.33 \\
\hline \hline
\end{tabular}

Propagation environment of type $r$, termed as $P T_{r}$, represent the channel environment of multicast users. $P T_{1}$ and $P T_{4}$ are suburban environments whereas $P T_{2}$ and $P T_{3}$ are wooded environment. $P T_{1}$ is in higher elevation angle compared to $P T_{4}$. The durations of non-fade (LOS) and fade for all propagation types are controlled by parameters $\beta, \chi, \alpha$ and $\delta$ in Table I. From [4], the duration of the open area LOS in suburban environments decrease slightly with decreasing elevation angle while for $P T_{2}$ and $P T_{3}$, which are wooded environment, the durations of open area state are significantly smaller than in suburban areas. The mobility level for all users is fixed throughout the multicast session. From the fixed mobility, the (non)fade duration in seconds are found to be larger than one RTT. In other words, the value of reported CSI remains constant during update and transmission period.

\section{ChanNel-Aware Scheduling Algorithm}

The considered scenario is a point-to-multipoint network between a satellite and $N$ direct receivers. A file of size $F$ is divided into $D$ number of segments. Each segment $p$ is fixed to $L$ bits. Prior to downlink transmission for each segment $p$, the $\mathrm{RNC}$ shall decide whether to delay or to transmit the segment according to the collected CSI parameters from all users. At this point it is proposed that the most recent Channel State Information (CSI) from all users in the multicast group is periodically collected. The CSI parameter, which is the segment error rate for user $j$, termed as $P_{0 j}$, from all users in the group is updated periodically. CSI information is time varying and uncorrelated among the users in the group. From this input, probability of retransmission for segment $i$ at frame $n$ for users $j=\{1, \ldots, N\}$ is calculated:

$$
\hat{P}_{R t x_{i}}(n)=1-\prod_{j=1}^{N}\left(1-P_{o j}(n)\right)
$$

and compared with QoS parameter $P_{0}$ which can be regarded as a tolerable upperbound segment error rate for any particular user $j$ in the form of upperbound probability of retransmission

$$
P_{T}=1-\left(1-P_{0}\right)^{N}
$$

The value of $\hat{P}_{R t x}$ is compared with $\left(M \times P_{T}\right)$ where $M$ is a design parameter. Based on this comparison, if the estimated $\hat{P}_{R t x}$ for segment $i$ at frame $n$ is less than $\left(M \times P_{T}\right)$,

$$
\hat{P}_{R t x} \leq M \times P_{T}
$$

then the segment $i$ will be transmitted. Otherwise, the segment will be delayed for 1 slot to wait for relatively good CSI parameter for all users.

To find optimum value of $M$, referred as $M_{O p t}$, simulation results of File Transfer Delay (FTD) against $M$ is analysed. $M_{O p t}$ is recorded when FTD is minimum. FTD is defined as session delay measured from the first segment of the file assigned to the scheduler until the last (re)transmitted segment is successfully received by all users, including scheduling delay and retransmission delay. Note that in this definition, retransmissions of lost segments have to wait until after the end of current pass of segments as outlined by the higher layer transport protocol. The calculation of FTD is as below:

$$
F T D=\left((D+N S R) \times T_{S l o t}\right)+(k M a x \times R T T)+T_{S c h}
$$

where FTD is measured in seconds, $D$ is number of segments in a file, NSR is total number of segments retransmitted throughout the session, $T_{\text {Slot }}$ is slot size in seconds, $k M a x$ is total number of retansmissions, $R T T$ is round-trip-time delay which is set to $800 \mathrm{~ms}$ and $T_{S c h}$ is scheduling delay measured in seconds. 


\section{CHANNEL-AwARE SCHEDULING IN HOMOGENEOUS ENVIRONMENT}

A scenario of homogeneous environment, where $N$ number of users in one propagation type throughout the multicast session are simulated. In Figure 2, propagation type 2 for $P_{0}=0.01$ is simulated, with defined channel parameters in Table I for $N=60$ and $D=800$ segments. From the figure, it is observed directly that the value of $M$ is 0.14 when FTD is minimum, i.e., $M_{O p t}=0.14$.

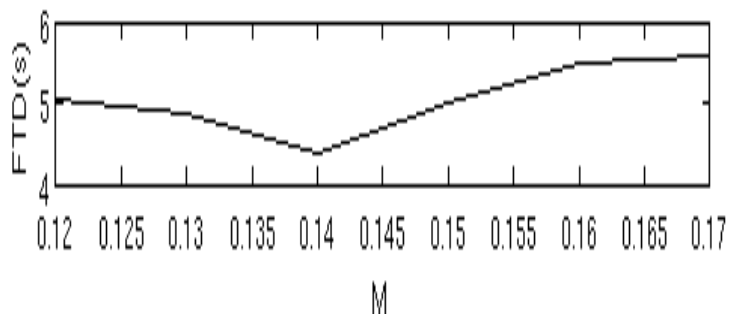

Fig. 2 Simulation result of FTD vs M

Results for other propagation types are tabulated and recorded in Table II. The value of $P_{0}$ is set to $P_{01}$ and $P_{02}$ taking the values of 0.01 and 0.001 , respectively. The four propagation types from Table I for $P_{0}=P_{02}$ are termed as Propagation Type 1, 2, 3, 4 and the same propagation types with $P_{0}=P_{01}$ are named as Propagation Type 5, 6, 7, 8. File size of 800 segments is chosen since it is noted that other file sizes give approximately the same values of $M_{O p t}$ although the FTD values increase with number of segments. For higher number of users, the values of $M_{O p t}$ and $\bar{P}_{R t x} / P_{T}$ is expected to increase as will be proven in numerical analysis later.

\begin{tabular}{|c|c|c|c|}
\hline \multirow{4}{*}{ 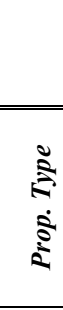 } & \multicolumn{2}{|c|}{ TABLE II VALUES OF $\xi, M_{O p t}$ AN } & $\frac{\bar{P}_{R t x}}{P_{T}}$ \\
\hline & \multicolumn{3}{|c|}{ File size $=800$ Segments } \\
\hline & & $\bar{P}_{R t x} / P_{T}$ & $\begin{array}{c}\xi= \\
M_{O p t} \\
\end{array}$ \\
\hline & $\begin{array}{c}M_{o p t} \\
\text { (from sim.) }\end{array}$ & $\begin{array}{c}\text { (from } \\
\text { calculation) }\end{array}$ & $\overline{\bar{P}}_{R t x} / P_{T}$ \\
\hline 1 & 0.72 & 0.72 & 1.00 \\
\hline 2 & 1.05 & 1.03 & 1.02 \\
\hline 3 & 0.20 & 0.20 & 0.99 \\
\hline 4 & 0.08 & 0.08 & 1.00 \\
\hline 5 & 0.19 & 0.19 & 1.01 \\
\hline 6 & 0.14 & 0.14 & 1.00 \\
\hline 7 & 0.05 & 0.05 & 1.03 \\
\hline 8 & 0.65 & 0.65 & 1.00 \\
\hline
\end{tabular}

To analyze $M_{O p t}$, the statistics of measured $P_{0 j}$ are recorded and it is observed that the value of $M_{O p t}$ is related to $\bar{P}_{R t x} / P_{T}$, where $\bar{P}_{R t x}=1-\left(1-\bar{P}_{0 j}\right)$ and $\bar{P}_{0 j}$ is average value of $P_{0 j}$ for a particular propagation type. Thus, equality

$$
M_{O p t}=\xi \times \bar{P}_{R t x} / P_{T}
$$

is formulated where $\xi$ is a coefficient to fit the linear relationship. From Table II, an important result is observed, where the value of $\xi$ is very close to 1 regardless of propagation types and file size.

From this observation, the linear equation is reduced to

$$
M_{O p t}=\frac{\bar{P}_{R t x}}{P_{T}}=\frac{1-\left(1-\bar{P}_{0 j}\right)^{N}}{1-\left(1-P_{0}\right)^{N}}
$$

where $\bar{P}_{0 j}=\frac{1}{N} \sum_{j=1}^{N} P_{0 j}$. From inequality (3) and equation (6), where the value of $M$ is taking $M_{O p t}$, it is noted that inequality (3) becomes

$$
\hat{P}_{R t x} \leq 1-\left(1-\bar{P}_{o j}\right)^{N}
$$

when $M_{O p t} \times P_{T}=1-\left(1-\bar{P}_{0 j}\right)^{N}$ where $1-\left(1-\bar{P}_{0 j}\right)^{N}$ on the right hand side of the inequality is the expected probability of retransmission. From Figure 3 the expected probability of retransmission increases with average $P_{0 j}$ and number of users $N$. In general, a multicast group with large number of users in relatively poor channel condition has a higher probability of retransmission. For this scenario, the scheduling algorithm shall delay the segment until the inequality is satisfied.

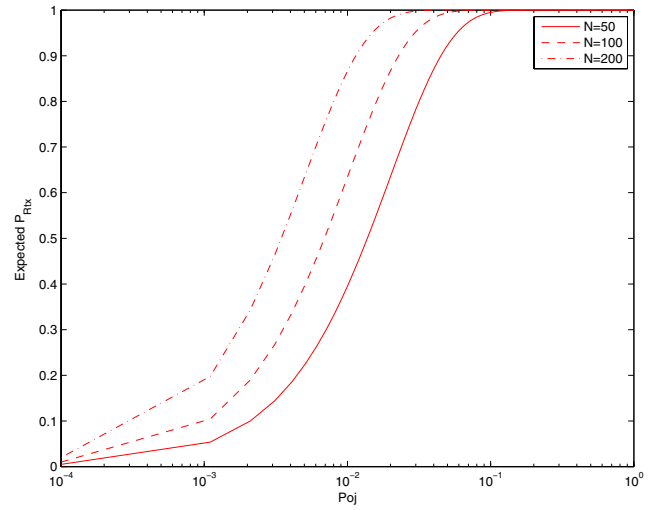

Fig. 3 The expected probability of retransmission vs $P_{0 j}$

V ChanNel-Aware Scheduling IN Mixed EnVironments

The algorithm in (7) is validated in mixed environments where users are spread around different propagation types. Three scenarios are simulated according to Table III where $N_{P T r}$ is 
number of users in terms of fraction from the total number of users, $N$. In scenario I, out of 100 users, each propagation type has $25 \%$ of them. In scenario II $40 \%$ of total users are in PT1, $10 \%$ in PT2, another $10 \%$ in PT3 and the last $40 \%$ in PT4. Whereas in scenario III, the propagation type mix is $10 \%$ in PT $1,40 \%$ in PT2, $40 \%$ in PT3 and $10 \%$ in PT4. From this simulation setup, file transfer delay (FTD) is again plotted against $M$ in Figure 4. In Table III, $M_{O p t}$ values from simulations and expected values from theoretical analysis are compared.

From the comparison of simulations and expected values, it is found that simulations error is within the range of $1 \%$ of theoretical value. Since the error is small it is concluded that the calculation to find $M_{O p t}$ in mixed environments is $M_{O p t}=\frac{\bar{P}_{R t x}}{P_{T}}=\frac{1-\left(1-\bar{P}_{0 j}\right)^{N}}{1-\left(1-P_{0}\right)^{N}}$, which is the same equation used in homogeneous environment.

TABLE III SimUlation SETUP AND RESUlts FOR MiXED ENVIRONMENTS SCENARIO

\begin{tabular}{|c|c|c|c|c|c|c|}
\hline Scenario & $\bar{N} N_{P T 1}$ & $\bar{~} N_{P T 2}$ & $\bar{N} N_{P T 3}$ & $\overline{N_{P T 4}}$ & $\begin{array}{l}M_{O P T} \\
\text { (from } \\
\text { sim.) }\end{array}$ & 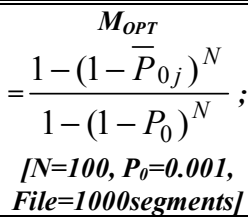 \\
\hline 1 & 0.25 & 0.25 & 0.25 & 0.25 & 0.7 & 0.69 \\
\hline 2 & 0.4 & 0.1 & 0.1 & 0.4 & 2.3 & 2.278 \\
\hline 3 & 0.1 & 0.4 & 0.4 & 0.1 & 0.6 & 0.660 \\
\hline
\end{tabular}

VI CSI COLLECTION POLICY

Looking at the CSI feedback collected at the gateway, it is observed that it is sufficient for the gateway to track the CSI only when there are changes. A change detection scheme based on sliding window is implemented to overcome the problem of high volume of feedback on the return link due to CSI updates. Furthermore, it avoids scheduling decision based on instantaneous short events which could lead to false alarm and receivers with large changes of CSI values to have higher priority to update their CSIs over other receivers. The choice of sliding window size, $L$, determines the robustness and agility of the algorithm against time-varying channel conditions. Therefore, it is essential that $L$ values should be chosen accordingly.

The change detection scheme is employed by implementing a loss function to filter the CSI variations. The loss function is chosen to be the least square over sliding window algorithm, termed as Windowed Least Square (WLS) which means the change detection algorithm uses time batches (sliding window) for averaging and thresholding. Note that in WLS there are two updates for each new sample, and a memory of the last $t-L$ measurements is needed for comparison. The first update is average value of $\gamma_{n}$ from an observation sliding window of size $L$. This average value, termed as $\hat{\theta}_{1}$ is compared with the second update which is an average value from a longer window, $\hat{\theta}_{0}$, using all past data since the last update, $t-L$. Parameter $\hat{\theta}_{1}$ is used as instantaneous value of $P_{0 j}$ and $\hat{\theta}_{0}$ is used to calculate $\bar{P}_{0 j}$.
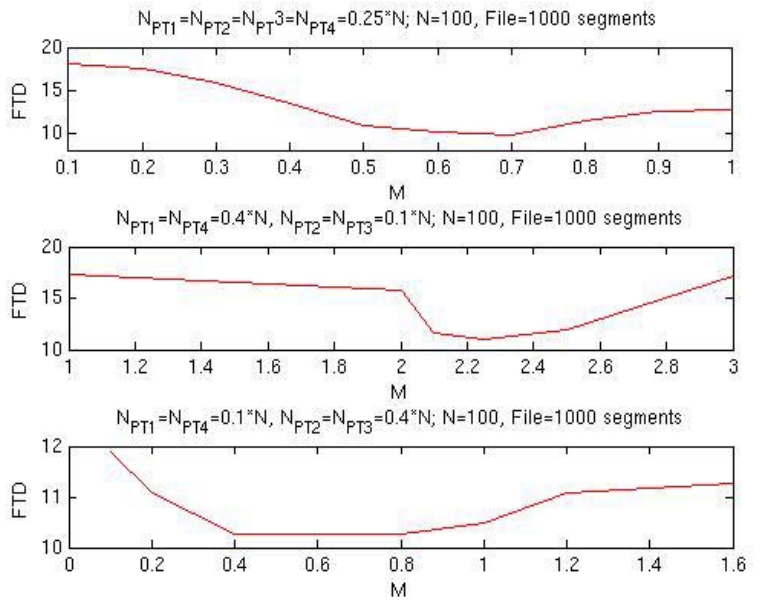

Fig. 4 FTD vs M for Mixed Environments

\begin{tabular}{|c|c|c|}
\multicolumn{2}{c|}{ TABLE IV CSI COLLECTION POLICY SETUP } \\
\hline Data & $\gamma_{1}, \gamma_{2}, \ldots . \gamma_{t-L}$, & $\gamma_{t-L+1,}, \ldots \ldots, \gamma_{t}$ \\
\hline Model & $\begin{array}{c}R_{0} \\
\text { Reference Model }\end{array}$ & $\begin{array}{c}R_{l} \\
\text { Test Model }\end{array}$ \\
\hline $\begin{array}{c}\text { WLS } \\
\text { Quantities }\end{array}$ & $\hat{\theta}_{0}$ & $\hat{\theta}_{1}$ \\
\hline Variance & $\lambda_{o}$ & $\lambda_{1}$ \\
\hline $\begin{array}{c}\text { Number of } \\
\text { Data }\end{array}$ & $n_{0}=t-L$ & $n_{l}=L$ \\
\hline
\end{tabular}

From this setup, a file of 100000 segments delivered to 50 multicast users is simulated in homogeneous environment. The result of normalized file transfer delay (FTD) against length of test window, $L$, is plotted in Fig 5. In Fig. 6, return link throughput (RLT) per user is plotted against $L$. RLT is defined as $\quad \mathrm{RLT}=\frac{U / F T D}{N}$ where $U$ is total number of updates in one multicast session.

From Fig 5 it is noted that FTD for users in suburban environment is higher than for users in wooded environment. This is because of the nature of suburban environment where the shadowed duration is longer than wooded environment. Note in the implemented scheduling algorithm, a longer shadowed duration would result segments to be delayed longer until good 
channel condition is reported. Also, it is observed that FTD increases with $L$. This is because for a fixed $t$, a shorter test window would allow a longer size for reference window. With a longer reference window, global parameter $\bar{P}_{0 j}$ is obtained from a larger sample size, where its value should converge to the theoretical value. This leads to optimum value of global parameter $\bar{P}_{0 j}$ in the right hand side of the inequality $1-\prod_{j=1}^{N}\left(1-P_{0 j}\right) \leq 1-\left(1-\bar{P}_{0}\right)^{N}$. In essence, a longer reference window (hence shorter test window) gives lower FTD.

In Fig 6, it is observed that RLT decreases with $L$. This is because in shorter test window, the discrepancy between test window and reference window is large. As $L$ increases, the variance of test and reference windows approaches a common value hence lower number of updates is observed. In general, a shorter test window results higher return link throughput compared to longer test window. From the two observations, it is essential to find $L_{O p t}$, where both FTD and RLT are low. Based on this criterion, the simulation results show that $L_{O p t}$ equals to 16 .

\section{CONCLUSION}

In this paper channel-aware scheduling for reliable transmission of multicast data over geostationary satellite networks is proposed. We introduce design parameter $M_{O p t}$ which reduces file transfer delay (FTD) by comparing most current values of CSI parameters to its expected values. Performance parameter FTD is observed in homogeneous and mixed environments. In both scenarios, simulations results to find $M_{O p t}$ are confirmed with theoretical results using expected values of CSI parameter. In conclusion, lowest FTD is achieved when estimated probability of retransmission is lower than its expected value. Note that the estimated probability of retransmission is calculated from instantaneous values of most recently reported CSI parameter and its expected probability is calculated from mean value of CSI parameter.

The assumption of periodic CSI collection from all users could be resource consuming in the return link. Thus, we adopt CSI collection policy where CSI values arrive intermittently or from only a subset of users. Instead of updating every slot, the change detection based on sliding window policy averages two CSI values from test and reference windows over two different lengths. The length of test window is denoted as $L$ and the length of reference window is $(t-L)$. If there are differences in the mean and variance values from the two windows, the CSI values from that particular user is updated. It is observed that a shorter test window gives lower FTD due to higher number of updates. We are still, however, use simplified assumptions on upper layer transport protocol. It will be a complete implementation if the scheduling and CSI collection algorithms are integrated with full implementation of reliable multicast transport protocol.

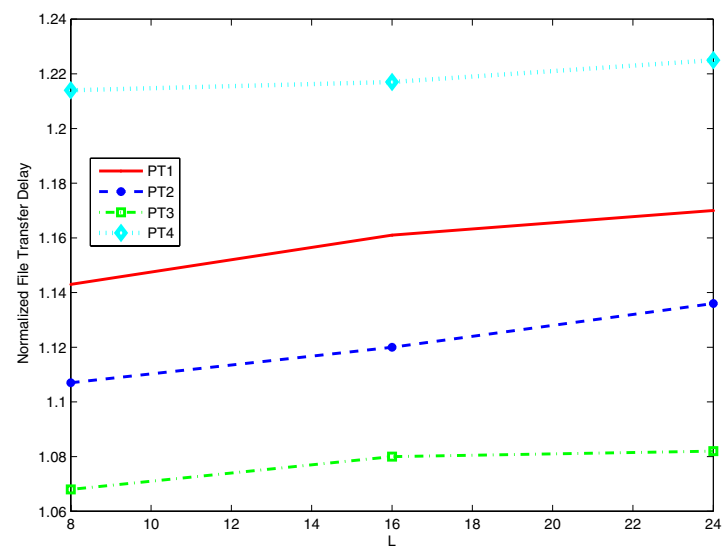

Fig 5 Normalized file transfer delay vs L

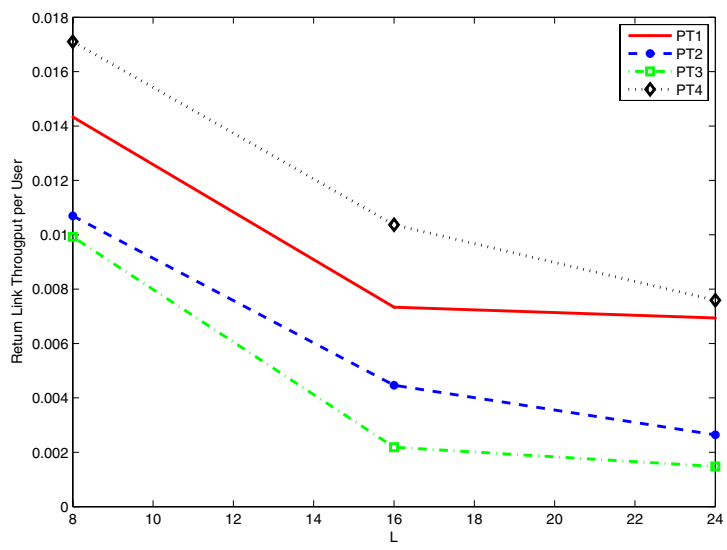

Fig. 6 Return link throughput vs L

\section{REFERENCES}

[1] J. Zhang, "Bursty traffic meets fading: a cross-layer design perspective," Proceeding of the 40th Allerton Conference on Communications, Control, and Computing, Oct. 2002.

[2] M.A.Haleem, R.Chandramouli, 'Adaptive Downlink Scheduling and Rate Selection: A Cross-Layer Design', IEEE Journal in Selected Areas in Communications, Vol. 23, No. 6, May 2005.

[3] Q.Liu, S.Zhou, G.B.Giannakis, 'Cross-Layer Scheduling With Prescribed QoS Guarantees in Adaptive Wireless Networks', IEEE Journal in Selected Areas in Communications, Vol. 23, No. 5, May 2005.

[4] L. E. Braten, T.Tjelta, 'Semi-Markov Multistate Modeling of Land Mobile Propagation Channel for Geostationary Satellites'. IEEE Transactions on Antennas and Propagation, Vol. 50, No. 12, Dec 2002.

[5] K. Miller and K. Robertson, "StarBurst Multicast File Transfer protocol (MFTP) Specification," IETF, IETF-Draft $<$ draft-miller-mftp-spec-03.txt $>$, July 1998.

[6] G. Giambene, S. Kota, 'Cross-Layer Protocol Optimization for Satellite Communications Networks: A Survey', International Journal of Satellite Communications and Networking, 2006, 24:323-341

[7] M. W. Koyabe, G. Fairhurst, 'Reliable Multicast Via Satellite: A Comparison Survey and Taxonomy', International Journal of Satellite Communications, 2001, 19:3-28 [8] F. Gustafsson, 'Adaptive Filtering and Change Detection', John Wiley\&Sons, 2000, pp221-223, 133-163 